\title{
KNOWLEDGE REGARDING CARE OF LOW BIRTH WEIGHT NEONATES AMONG NURSES OF MORANG, NEPAL
}

Anuja Kachapati, ${ }^{1}$ Kavita Lamichhane, ${ }^{1}$ Sangam Shrestha ${ }^{2}$

\section{ABSTRACT}

\section{INTRODUCTION}

Babies born preterm or at low birth weight are at increased risk of immediate life-threatening health problems as well as long-term complications and developmental delays. The occurrence of this condition causes substantial morbidity and mortality in children. The nurses are the key persons to provide care for the low birth weight neonates. The role of nurses to assess the low birth weight babies and to protect them from various complications.

\section{MATERIAL AND METHODS}

A descriptive cross-sectional design was used to find out the level of knowledge regarding care of low birth weight neonates among 60 registered nurses of Nepal (staff nurses and bachelor nurses) by using enumerative technique in Koshi Hospital, Biratnagar, Nepal. Semi-structured, pre-tested interview schedule was used to collect data and analysed by using descriptive and inferential statistic with SPSS version 16.

\section{RESULTS}

The study findings revealed that $63.3 \%$ of the respondents had high level of knowledge regarding care of low birth weight neonates. There was no statistically significant association between respondents' professional qualification and respondents' level of knowledge regarding care of low birth weight neonates.

\section{CONCLUSION}

The study concluded that more than half of the respondents had high level of knowledge regarding care of low birth weight neonates. Nurse is an important primary care provider and therefore, her education and access to information on care of low birth weight new born will help her provide adequate care and prevent complications in low birth weight babies.

\section{KEYWORDS}

Knowledge, Low birth weight neonates, Nurses,

1. Universal College of Nursing Sciences, (UCMS) Bhairahawa, Nepal

2. Nursing Officer, Koshi Zonal Hospital, Biratnagar, Nepal

DOI: http//doi.org/10.3126/jucms.v9i01.37983

For Correspondence

Ms. Anuja Kachapati

Universal College of Nursing Sciences

UCMS, Bhairahawa, Nepal

Email:kachapatianuja@gmail.com 


\section{INTRODUCTION}

Low birth weight (LBW) is defined as a birth weight of a live born infant of 2,499 $\mathrm{g}$ or less, regardless of gestational age. Subcategories include very low birth weight (VLBW), which is less than $1500 \mathrm{~g}$, and extremely low birth weight (ELBW), which is less than $1000 \mathrm{~g}$.

Globally, 2.5 million children died in the first month of life at an average rate of 18 deaths per 1,000 live births in 2017. There are approximately 7,000 new born deaths every day, accounting for about $47 \%$ of all child deaths. Globally, the number of neonatal deaths declined from 5 million in 1990 to 2.5 million in $2017 .^{2}$

Low birth weight and preterm birth are the most important indirect cause of new born mortality. Globally, the prevalence rate of preterm babies is 15 million and low birth weight babies are approximately 20 million, among them $96.5 \%$ low birth weight occur in developing countries. Usually prematurity and low birth weight occur together, both carrying a high birth rate of morbidity and mortality unless optimal care is given to maintain life.

The prevalence of LBW in Nepal was $12 \%$ which was calculated from the available birth weight of infants. There was no change in the percentage of babies with a low birth weight between 2011 and 2016 in the country. ${ }^{4}$ Similarly, the prevalence of LBW was $15.4 \%$ in a data base survey in Nepal. ${ }^{5}$ As per the study conducted in Nepal different hospitals, the prevalence of LBW in Dhulikhel Hospital the incidence of LBW was $11.07 \%,{ }^{6} 23.1 \%$ Koshi Zonal Hospital ${ }^{7}$ and $11.99 \%$ in Patan respectively.

Birth weight is an important indicator of a population's health. In 2010, a total of 10.9 million births were preterm and appropriate-for-gestational age, 29.7 million births were full term and small-for-gestational age (SGA), and 2.8 million births were preterm and SGA among the total 135 million births in developing countries. Seventy-two percent of LBW infants in developing countries are born in Asia. In Nepal, LBW neonates comprised of $21.81 \%$ in $2016 .{ }^{8}$

Due to lack of immunity and LBW new born are at high risk of having problem with increased chance to acquire infection which later on can lead to death due to lack of immunity. The LBW new born have face may problems like hypothermia, feeding difficulties and hypoglycaemia. Low birth weight babies are immature, and they need special nursing care.

LBW/premature babies are at greater risk of illness and death is that they lack the ability to control their body temperature, i.e., they get cold or hypothermic very quickly. A cold new born stops feeding and is more susceptible to infection. Kangaroo Mother Care (KMC) is the early, prolonged, and continuous skin-to skin contact between the mother (or substitute) and her low birth weight infant, both in hospital and after early discharge, until at least the $40^{\text {th }}$ week of postnatal gestation age, with ideally exclusive breastfeeding and proper follow-up. Kangaroo Mother Care (KMC) is a powerful and easy to use method to promote the health and well-being of low birth weight (LBW). ${ }^{10}$

Since the birth weight is the most important factor determining the survival chances of neonates, nurses must have up to date knowledge regarding the care of the low birth weight babies. With this background the study was undertaken with the objectives were to find out the knowledge regarding care of low birth weight neonates among nurses and to find out association between the level of knowledge regarding care of low birth weight neonates with a selected socio-demographic variable.

\section{MATERIAL AND METHODS}

A descriptive cross-sectional research design was conducted to find out the level of knowledge regarding care of low birth weight neonates among 60 registered nurses of Nepal (staff nurse and bachelor nurse) by using enumerative sampling technique in Koshi Hospital of Biratnagar, Morang district, Nepal. Semi structured interview schedule was used for data collection within one-month period during the month of August, 2020. A descriptive statistics and inferential statistics (chi-square tests) were used to find out the association between level of awareness and their socio-demographic variables with SPSS version 16.

Ethical approval was obtained from the Institutional Review Committee (IRC) with the reference number UCMS/IRC /175 /19 of Universal College of Medical Sciences, Bhairahawa and administrative approval was obtained from the concerned authorities prior to data collection. Written informed consent was obtained from each respondent by clarifying the objective of the study.

\section{RESULTS}

More than half $(56.7 \%)$ of respondents had completed Proficiency Certificate Level in Nursing and $43.3 \%$ had completed Bachelor in Nursing. Twenty percent of respondents were working in medical ward as well as in maternity ward and only $3.3 \%$ were working in orthopaedic ward. Nearly half of respondents (41.7\%) had 5-10 years of working experience whilst $11.7 \%$ had more than 15 years of experience. Regarding training, $26.7 \%$ of respondents had received training on care of low birth weight neonates.

Regarding meaning of LBW, VLBW and ELBW neonates, $95 \%, 88.3 \%$ and $93.3 \%$ of respondents answered as neonates weighing less than 2500 grams, less than 1500 grams and less than 1000 grams respectively. 
Table 1. Respondents' knowledge regarding risk factors and major aspects of care for $L B W$ neonates $(n=60)$

\begin{tabular}{lcc}
\hline Variables & Frequency & .Percentage \\
\hline Risk factors of LBW** & & \\
Young maternal age* & 51 & 85.0 \\
Small maternal body structure* & 19 & 31.7 \\
Hyperemesis gravidarum* & 22 & 36.7 \\
Preterm labour* & 54 & 90.0 \\
Less intake of nutritious diet* & 50 & 83.3 \\
Inadequate antenatal visits* & 22 & 36.7 \\
Antepartum hemorrhage* & 22 & 36.7 \\
Previous home delivery & 3 & 5.0 \\
Mother's physical disability & 17 & 28.3 \\
History of maternal infections in previous pregnancy & 2 & 3.3 \\
Low socio-economic status* & 42 & 70.0 \\
Major aspects of care for LBW neonates** & & \\
Maintenance of thermoregulation* & 55 & 91.7 \\
Promotion of sleep & 15 & 25.0 \\
Prevention of infection* & 51 & 85.0 \\
Maintenance of breathing* & 33 & 55.0 \\
Maintenance of nutrition and hydration* & 53 & 88.3 \\
Kissing the baby for gentle stimulation & 5 & 8.3 \\
Administration of BCG vaccine & 12 & 20.0 \\
\hline
\end{tabular}

**Multiple responses *Correct response

Mean percentage score of risk factors LBW:58.76

Mean percentage score of major aspects of care for LBW neonates: 80

Most of the respondents $(90 \%)$ knew preterm labour as risk factors of LWB and $91.7 \%$ answered maintenance of thermoregulation as major aspects of care for LBW neonates.

Table 2. Respondents' knowledge regarding hypothermia in $\mathrm{LBW}$ neonates $(\mathrm{n}=60)$

\begin{tabular}{lcc}
\hline Variables & Frequency & Percentage \\
\hline Meaning of hypothermia & & \\
Body temperature less than $35.5^{\circ} \mathrm{C}$ & 18 & 30.0 \\
Body temperature less than $36.5^{\circ} \mathrm{C}^{*}$ & 20 & 33.3 \\
Body temperature less than $37.5^{\circ} \mathrm{C}$ & 9 & 15.0 \\
Body temperature less than $34.5^{\circ} \mathrm{C}$ & 13 & 21.7 \\
Methods of prevention of hypothermia in LBW & & \\
neonates** & 32 & 53.3 \\
Warm chain* & 7 & 11.7 \\
Warm compress & 57 & 95.0 \\
Kangaroo mother care* & 1 & 1.7 \\
Bathing with warm water immediately & 49 & 81.7 \\
Keeping neonate in pre-warmed radiant warmer and & & \\
incubator* & & \\
\hline
\end{tabular}

*Correct response $* *$ Multiple responses

Mean percentage score of methods of prevention of hypothermia in

LBW neonates:76.67

Less than half of respondents (33.3\%) answered meaning of hypothermia as body temperature less than $36.5^{\circ} \mathrm{C}$. Majority of respondents $(95 \%)$ answered kangaroo mother care as preventive method of hypothermia in LBW neonates.

All of the respondents answered KMC as a method of keeping the baby warm through continuous skin to skin contact with the mother. More than half of respondents (63.3\%) answered skin to skin contact, breastfeeding and support for mother and baby as components of $\mathrm{KMC}$ whereas $71.7 \%$ of respondents answered KMC should be continue until the baby gain weight of $2.5 \mathrm{~kg}$. Majority of respondents (85\%) answered that family members can be involved in KMC.

Table 3. Respondents' knowledge regarding feeding for LBW neonates $(n=60)$

\begin{tabular}{|c|c|c|}
\hline Variables & Frequency & Percentage \\
\hline \multicolumn{3}{|l|}{ Best choice of nutrition } \\
\hline Exclusive breastfeeding* & 54 & 90 \\
\hline \multicolumn{3}{|l|}{$\begin{array}{l}\text { Methods of maintaining nutrition and } \\
\text { hydration if not able to be breastfed** }\end{array}$} \\
\hline Cup and spoon feeding* & 45 & 75.0 \\
\hline Orogastric feeding* & 24 & 40.0 \\
\hline \multicolumn{3}{|l|}{ Calorie requirement during $1^{\text {st }}$ week of life } \\
\hline $60 \mathrm{kcal} / \mathrm{kg} /$ day* & 31 & 51.7 \\
\hline \multicolumn{3}{|l|}{ Fluid requirement } \\
\hline $50-100 \mathrm{ml} / \mathrm{kg} /$ day & 23 & 38.3 \\
\hline $60-100 \mathrm{ml} / \mathrm{kg} /$ day* & 19 & 31.7 \\
\hline $70-100 \mathrm{ml} / \mathrm{kg} /$ day & 10 & 16.7 \\
\hline $60-120 \mathrm{ml} / \mathrm{kg} /$ day & 8 & 13.3 \\
\hline \multicolumn{3}{|l|}{ Time of feeding } \\
\hline 2-3 hourly* & 38 & 63.3 \\
\hline \multicolumn{3}{|l|}{ Position after feeding } \\
\hline Supine & 6 & 10.0 \\
\hline Left side & 35 & 58.3 \\
\hline Right side* & 14 & 23.4 \\
\hline Prone position & 5 & 8.3 \\
\hline \multicolumn{3}{|l|}{ NICU Environment $(n=54)$} \\
\hline $\begin{array}{l}\text { Professional and public/visitor traffic should } \\
\text { be same }\end{array}$ & 6 & 11.1 \\
\hline Warm* & 52 & 96.3 \\
\hline Proper light* & 44 & 81.5 \\
\hline Provided with 2 visitors of each baby & 2 & 3.7 \\
\hline Free from excessive sound* & 37 & 68.5 \\
\hline
\end{tabular}

**Multiple responses *Correct response

Mean percentage score of NICU environment to be maintained:80.57

Majority of respondents (90\%) answered exclusive breastfeeding as best nutrition for LBW neonates. More than half of respondents (75\%) knew that cup and spoon feeding should be done if LBW neonates are not able to breastfed. Likewise, regarding calorie requirement during first week of life, $51.7 \%$ answered $60 \mathrm{kcal} / \mathrm{kg} / \mathrm{day}$. Regarding fluid requirement, $38.3 \%$ answered $50-100 \mathrm{ml} / \mathrm{kg}$ /day whereas only $13.3 \%$ answered $60-120 \mathrm{ml} / \mathrm{kg} /$ day. More than half of respondents (63.3\%) answered time of feeding as 2-3 hourly and $58.3 \%$ knew LBW neonates should be place in right side after feeding.

More than half of the respondents $(65 \%)$ knew that multinutrients supplementation should be given for LBW neonates as they have inadequate stores of the most nutrients.

Majority of respondents (90\%) knew special care for LBW neonates should be provided in NICU.

Regarding environment in NICU for LBW neonates, $96.3 \%$ of 
respondents answered warm, $81.5 \%$ as proper light and $68.5 \%$ free from excessive sound.

Table 4. Overall respondents' knowledge regarding care of low birth weight neonates $(\mathrm{n}=60)$

\begin{tabular}{lcc}
\hline Level of knowledge & Frequency & Percentage \\
\hline High & 38 & 63.3 \\
Low & 22 & 36.7 \\
Total & 60 & 100 \\
\hline
\end{tabular}

Mean score-23.6 out of 32

Majority $63.3 \%$ of respondents had high knowledge and $36.7 \%$ had low knowledge regarding care of low birth weight neonates

Table 5. Association between respondents' professional qualification and respondents' level of knowledge regarding care of $L B W$ neonates

\begin{tabular}{|c|c|c|c|c|}
\hline \multirow{2}{*}{$\begin{array}{l}\text { Professional } \\
\text { qualification }\end{array}$} & \multicolumn{2}{|c|}{ Level of knowledge } & \multirow[t]{2}{*}{$\chi^{2}$} & \multirow[t]{2}{*}{ p-value } \\
\hline & High & Low & & \\
\hline PCL & $23(22.2)$ & $13(13.8)$ & 0.188 & 0.665 \\
\hline B.N. & $14(14.8)$ & $10(9.2)$ & & \\
\hline
\end{tabular}

Significance level at 0.05

There is no statistically significant association between respondents' professional qualification and respondents' level of knowledge regarding care of LBW neonates $(p=0.665)$.

\section{DISCUSSION}

The study findings showed $70 \%$ of the respondents had knowledge that low socio-economic status is a risk factor of LBW which is not consistent with the study of Amoula et al (2016) conducted in NICU at Soba University Hospital, Sudan which shows $62 \%$ had knowledge that low socioeconomic status is a risk factor of LBW. ${ }^{11}$

Similarly, the findings showed $85 \%$ of the respondents had knowledge that young maternal age is a risk factor of LBW which is not consistent with the study of Levision et al (2014) ${ }^{12}$ conducted in Malawi shows $64.3 \%$ had knowledge that young pregnancy is a risk factor of LBW. This variation might be due to sample size.

The results revealed $91.7 \%$ and $85 \%$ of the respondents had knowledge that maintenance of thermoregulation and prevention of infection are the major aspects of care for LBW neonates respectively which are not consistent with the study of Ayiasi et al (2014) ${ }^{13}$ conducted in Masindi, Uganda which shows $79.2 \%$ and $72.1 \%$ had knowledge about maintenance of thermoregulation and infection prevention as the major aspects of care for LBW neonates. This variation might be due to sample size.
The results showed $55 \%$ of the respondents had knowledge that maintenance of breathing is a major aspect of care for LBW neonates which is inconsistent with the study of Amoula et al (2016) ${ }^{11}$ conducted in NICU at Soba University Hospital, Sudan which shows that $90 \%$ of the respondents had knowledge that maintenance of breathing is a major aspect of care for LBW neonates.

The results showed $33.3 \%$ of the respondents had knowledge regarding meaning of hypothermia. The study findings showed $53.3 \%$ of the respondents had knowledge that warm chain is a method of prevention of hypothermia which is consistent with the study of Ayiasi et al (2014) which shows $54 \%$ of the respondents had knowledge that warm chain is a method of stabilizing the temperature of LBW neonates. ${ }^{13}$

The study findings showed $81.7 \%$ of the respondents had knowledge that keeping neonates in pre-warmed radiant warmer and incubator is a method of prevention of hypothermia which is contradicted with the study of Phanase (2016) conducted at Hospital of Vidarbha which shows that $42.2 \%$ had knowledge that keeping neonates in pre-warmed radiant warmer and incubator is a method of prevention of hypothermia. ${ }^{14}$

The study findings showed $95 \%$ of the respondents had knowledge that $\mathrm{KMC}$ is a method of prevention of hypothermia which is inconsistent with the study of Ayiasi et al (2014) conducted in Masindi, Uganda which shows that $72.1 \%$ of the respondents had knowledge that $\mathrm{KMC}$ is a method of prevention of hypothermia in LBW neonates. ${ }^{13}$

The findings showed that $85 \%$ of the respondents had knowledge that family members can be involved in KMC which is inconsistent with the study of Dalal et al (2013) conducted in Ahmedabad district which shows that $31.7 \%$ had knowledge that KMC can be given at home. ${ }^{10}$

The study findings showed all of the respondents had knowledge that it is necessary to wash hands before and after touching each baby. A comparative study was conducted by Capretti MG, Sandri F (2008) ${ }^{15}$ in Italy to assess the impact of a standardized hand hygiene program on the incidence of nosocomial infection in very low birth weight infants among staff nurses working in neonatal intensive care unit and the findings showed that the incidence of nosocomial infection was significantly reduced after the introduction of a standardized hand washing protocol among in very low birth weight infants.

There was no statistically significant association between respondents' socio demographic data and level of knowledge regarding care of LBW neonates ( $p=0.665)$. It is supported by study conducted in Vidarbha region, Maharashtra which reveals there is no significant association found between 
knowledge and the demographic variables of the participants. ${ }^{15}$

\section{CONCLUSION}

Based on the study findings, it is concluded that more than half of the respondents have high level of knowledge regarding care of low birth weight neonates. They have high knowledge regarding meaning of LBW, VLBW and ELBW neonates, risk factors and major aspects of care for LBW neonates, methods of prevention of hypothermia in LBW neonates, KMC (meaning, components, continuation, advantages and methods, involvement of family members in KMC), maintenance of nutrition and hydration (best choice of nutrition, methods, reasons for multinutrient supplementation, time of feeding), ward for special care and infection prevention of LBW neonates. Still they have low knowledge regarding meaning of hypothermia, duration of KMC, fluid requirement, duration of multi-nutrients supplementation and positioning after feeding of LBW neonates. There is no statistically significant association between respondents' professional qualification and respondents' level of knowledge regarding care of LBW neonates.

\section{LIMITATION OFTHE STUDY}

The study was conducted among sixty nurses in a hospital of Morang district. So, findings of the study cannot be generalized in other setting.

\section{ACKNOWLEDGEMENT}

Researchers deeply express their heartfelt thanks to all who participated in the study for immense support and cooperation. Researchers express their deep and sincere gratitude to all those experts for their valuable judgment, constructive feedbacks and enlightening suggestions throughout the study.

\section{REFERENCES}

1. UNICEF. Fact sheet. Low birth weight. Retrieved from: https://data.unicef.org/topic/nutrition/low-birthweight/

2. World Health Organization, Fact sheet. Newborns: Reducing Mortality, World Health Organization, 2018.

3. WHO. (2017). Maternal, newborn, child and adolescent health. February 14, 2017. Retrieved from http:/www.who.int/ maternal_child_adolescent/topics/newborn/care_of_preterm/en/

4. Ministry of Health, Nepal; New ERA; and ICF. 2017. Nepal Demographic and Health Survey 2016. Kathmandu, Nepal: Ministry of Health, Nepal.
5. Singh U, Ueranantasun A, Kuning M. Factors associated with low birth weight in Nepal using multiple imputation. BMC Pregnancy and Childbirth. 2017;17:67.

6. Singh S, Shrestha S, Marahatta S. Incidence and risk factors of low birth weight babies born in Dhulikhel Hospital. Journal of Institute of Medicine Nepal. 2010;32(3):39-42.

7. Malla M, Joshi DR, Chhetri K, Pandey P. Prevalence and contributing factors of low birth weight babies in institutional delvery. J Patan Acad Heal Sci. 2015;2(1):26-9.

8. Koirala AK, Bhatta DN. Low-birth-weight babies among hospital deliveries in Nepal: a hospital-based study. Int J Womens Health. 2015;7:581-5.

9. Singh S, More M, Kulkarni P, More P. A study to assess the knowledge regarding care of low birth weight baby among mothers on selected hospitals of Pune city. IJAR. 2019;5(3):813.

10. Dalal A, Bala DV, Chauhan S. A cross-sectional study on knowledge and attitude regarding kangaroo mother care practice among health care providers in Ahmedabad District. International Journal of Medical Science and Public Health. 2014 Mar;3(3):253-7.

11. Amoula IW, Kambal E.I. Pediatric nurse's knowledge and practices regarding nursing management of premature babies in neonatal intensive care unit. International Journal of Recent Research in Life Sciences. 2016;3(4):1-9.

12. Levison J, Nanthuru D, Chiudzu G, Kazembe PN, Phiri H, Ramin SM, Aagaard KM. Qualitative assessment of attitudes and knowledge on preterm birth in Malawi and within country framework of care. BMC Pregnancy and Childbirth. 2014 Dec;14:123.

13. Ayiasi RM, Criel B, Orach CG, Nabiwemba E, Kolsteren P. Primary healthcare worker knowledge related to prenatal and immediate newborn care: a cross sectional study in Masindi, Uganda. BMC Health Services Research. 2014 Dec;14(1):65.

14. Phanase BN. Effectiveness of structured teaching programme on knowledge regarding care of low birth weight babies among staff nurses. International Journal of Nursing Education and Research. 2016;4(2):95-8.

15. Capretti MG, Sandri F. Tridapalli E, Galletti S, Petracci E, Faldella G. Impact of a standardized hand hygiene program on the Incidence of nosocomial infection in very low birth weight infants. Am J Infect Control. 2008 Aug;36(6):430-5. 